\title{
SPRING-8 BEAMLINE CONTROL SYSTEM
}

\author{
T. Ohata, T. Fukui, Y. Furukawa, M. Ishii, M. Kodera, T. Masuda, T. Matsushita, \\ T. Nakatani, M. Takeuchi, K. Tamasaku, R. Tanaka and A. Yamashita, \\ SPring-8, Hyogo, Japan
}

\begin{abstract}
A third generation light source, SPring-8, has been providing high brilliant $\mathrm{X}$-ray beams to experimental users. Beamline control system in the SPring- 8 is designed to achieve the independent tuning of insertion devices by beamline users. The system is designed adopting the same control framework of the SPring-8 storage ring in order to realize smooth operation between facilities. Beamline users control the equipment by sending control commands to a server process which is running on a beamline-managing computer. The communication is performed either by serial line or TCP/IP sockets over Ethernet. Introducing firewall systems to the beamline user's network protects the beamline network security. The secure network guarantees both safety equipment controls at higher level and realizes data transfer from on-site data taking machine to off-site computers via network. In this paper, we give the basic design and present an outline of the beamline control system at SPring-8.
\end{abstract}

\section{SYSTEM DESIGN}

In SPring-8, the beamline control system supports a front-end, an X-ray transport channel, an interlock system and an insertion device (ID) [1]. Beamline users are able to control all of the beamline components by the same software framework which is provided by the control group. The beamline users may use a system of their choice for the experimental stations. The beamline control architecture is designed to realize the independent tuning of insertion devices by beamline users, which allows flexible operation of IDs without interference to the storage ring (SR) operation.

The beamline system is designed using the control framework of the SPring-8 storage ring [2] which is built using the so-called Standard Model concept.

\subsection{Conceptual design}

At SPring-8, the IDs are considered a part of the beamline, so the beamline users can change the gap of the IDs as a tunable parameter. It is one of the specific

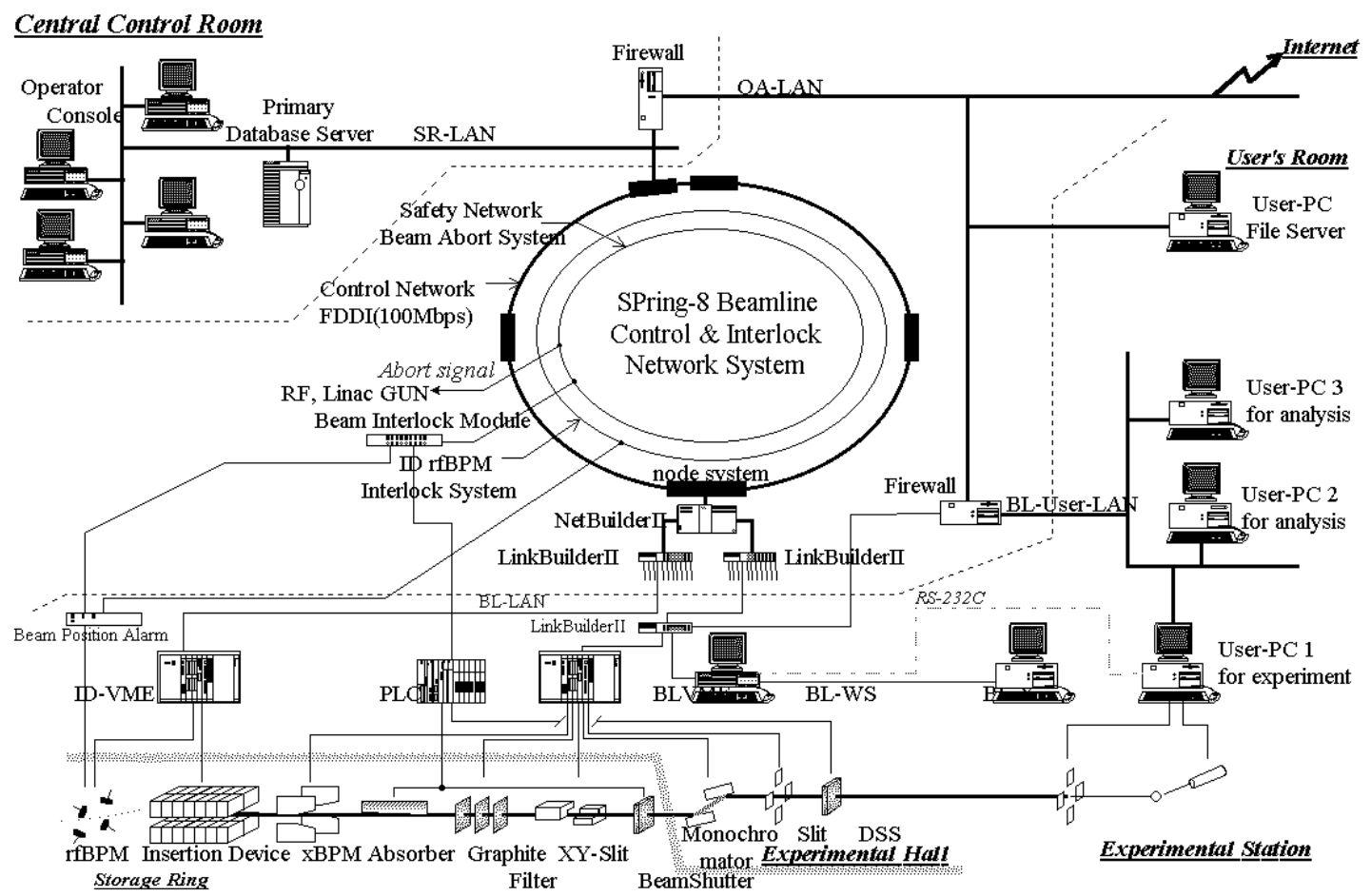

Figure 1. Schematic diagram of the beamline control system and network with firewall 
features of the SPring- 8 as a third-generation light source facility.

The control system includes the beamline interlock system (BLIS) for protection of the beamline equipment. Human safety is guaranteed by a safety interlock system. The BLIS monitors components such as beam shutter, vacuum gauge, cooling water temperature and the status of hatches. BLIS is designed to be highly safe and simple to operate. The design concept of the beamline control is summarized as follows:

(1) Smooth integration of the interlock and the control system.

(2) Manage all the information through the network with high security.

(3) Sophisticated software design for easy maintenance and user orienting.

(4) GUI-based applications with unified look \& feel.

\subsection{Computer and data acquisition systems}

The computing system of a beamline consists of a beamline-managing computer (BL-WS), experimental user's computers, and VME-based board computers (BLVME, ID-VME). As shown in Figure 1, a typical beamline computing system has four computers as BLWS (HP9000/B132), BL-VME (HP9000/743rt), BL-X (PC compatible UNIX) and ID-VME (HP9000/743rt). A programmable logic controller PLC (Mitsubishi Q2Aseries) is also installed at each beamline. The BL-WS and BL-VME manage all beamline components. The beamline operation terminal (BL-X) is connected to the BL-WS, which is prepared for an experimental user. Several controllers such as ID gap controller or rfBPM controller are managed by the ID-VME. A PLC, which are weakly linked to each other using the network, is used as the core system of the BLIS. A RS232C serial line is used to communicate between the BL-WS and the PLC for data acquisition from the BLIS. The same data acquisition software, poller/collector of the storage ring [3], takes all of the beamline data such as ID gap and valve status and other information. The beamline data is also stored in the SPring- 8 database system managed by Sybase Adaptive Server Enterprise 11[3].

Two server processes on the beamline control workstation, request server (RS) and command interpreter (CI), are newly introduced to the control software framework. Figure 2 shows the software architecture

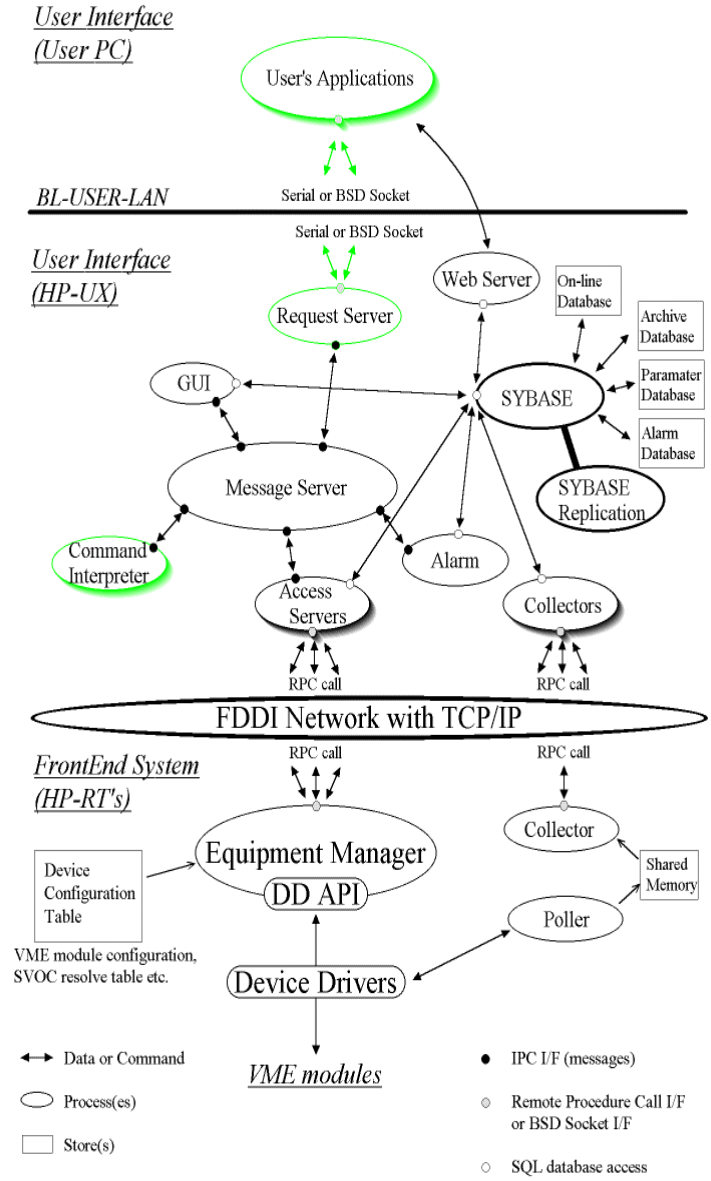

Figure 2: Schematic drawing of the software framework of SPring-8 control system.

with the RS and the CI. The RS is the server software, which manages user's command requests sent from either a serial line or the Ethernet. It accepts a high-level compound command and forwards it to the CI. The CI receives the compound SVOC message issued from user's machine or operation GUI then translates and decomposes it to a set of primitive SVOC messages. For example, it is used for the cooperative operation such as a monochromator and $\mathrm{x}$-ray energy. Table 1 shows an example of a monochromator control with the CI. A series of primitive messages are sequentially passed to the access server (AS) according to the control sequence. The introduction of the CI reduces the number of SVOC messages sent from user machine. In this framework, the

Table.1 : An example of the SVOC messages for monochromator control with the CI.

\begin{tabular}{|l|l|}
\hline Compound SVOC & put/bl_47in_tc1_stmono_1/\%fkev \\
\hline Primitive SVOC & put/bl_47in_tc1_stmono_1_theta_y1/\%dpulse \\
& put/bl_47in_tc1_stmono_1_theta_theta/\%dpulse \\
& put/bl_47in_tc1_stmono_1_theta_phi1/\%dpulse \\
& $\begin{array}{l}\text { put/bl_47in_tc1_stmono_1_theta_phi2/\%dpulse } \\
\text { Iteration with small pulse. }\end{array}$ \\
\hline
\end{tabular}


software overhead due to the network communication is substantially reduced, and the performance of communication between the user machine and the control workstation is improved remarkably.

\subsection{Network system}

There are three network zones around the firewall system, SR(BL)-LAN, BL-USER-LAN, and the public OA-LAN. Optical fiber Ethernet is used for backbone of the BL-USER-LAN with 100Mbps band-width. A RJ45 plug socket with 10BASE-T was supplied for each beamline. The firewall system is introduced to protect the network security at the SR(BL)-LAN and the BLUSER-LAN. A BL-USER-LAN in the experimental hall is a protected Demilitarized zone opened for experimental users. A laboratory public OA-LAN is connected to the internet directly via a router. The firewall systems are installed to interconnect those LANs with reasonable security and usability. The beamline users control beamline equipment safely using access control provided by the virtual LAN function. The experimental data can be sent out from the experimental hall to off-site home institutes via the network. The system is flexible enough to impose any suitable security rules and the network performance is satisfactory. We install a security policy such that no access from outside of the laboratory is allowed to reach the protected network zone.

\subsection{Interlock system}

The BLIS is constructed by hardwired relays and PLC interlock modules. Any software on WS or VME is not placed in the interlock sequence for better human safety. The BLIS status of the beamline is taken independently from each other to be able to support independent connection / disconnection of each interlock sub-system.

The new interlock system for beamline and rfBPM interlock system are introduced. The rfBPM interlock system consists of modules of beam position alarm (BPA), and gap alarm (GAPA). The BPA monitors the electron beam position near the insertion devices in the storage ring. The GAPA, connected by optical bus to each other, judge the abort condition by using several pieces of information such as storage ring current, gap status of whole insertion devices and the signal from the BPA. Whenever the electron beams shifts from the normal position under specific condition, the interlock system aborts the stored beam within $800 \mu \mathrm{sec}$.

\section{SUMMARY AND PLAN}

The beamline control system is highly functional and working quite stably in SPring-8. The independent tuning of IDs is satisfactory for experimental users. According to the user requirements, we are planning to upgrade the beamline network system to have higher throughput and wider bandwidth. The backbone of the network system will be upgraded from $100 \mathrm{Mbps}$ to $1 \mathrm{Gbps}$ this winter. The throughput of the data transfer will be greatly improved.

There is an announcement that the VME CPU board, HP9000/743rt, will not be available after this autumn. We have decided that it is necessary for the software framework to migrate to another CPU board, such as the Intel CPU family. We have already started the migration R\&D task to port the software framework to the new one with a new operating system.

\section{REFERENCES}

[1] T. Ohata et al., "SPring-8 beamline control system", J. Synchrotron Rad., 5, 1998, p.590.

[2] R. Tanaka et al., "Control System of the SPring-8 Storage Ring ", ICALEPCS'95, Chicago, USA, 1995, p.201.

[3] A. Taketani et al., "Data acquisition system with database at the SPring-8 storage ring", ICALEPCS'97, Beijing, China, 1997, p.437.

[4] A. Yamashita et al., "The database system for the SPring-8 storage ring control", ICALEPCS'97, Beijing, China, 1997, p.427. 